\title{
Perfil epidemiológico do atendimento antirrábico humano em Porto Alegre, RS, Brasil
}

\author{
Epidemiologic profile of human anti-rabies treatment \\ in Porto Alegre, RS, brazil
}

Rejane Dias Veloso ${ }^{1}$

Denise Rangel Ganzo de Castro Aerts ${ }^{1}$

Liane Oliveira Fetzer ${ }^{2}$

Celso Bittencourt dos Anjos ${ }^{3}$

José Carlos Sangiovanni ${ }^{4}$

${ }^{1}$ Programa de PósGraduação em Saúde Coletiva, Universidade Luterana do Brasil. Av. Farroupilha 8001/prédio 14/ sala 226, São José. 92425-900 Canoas RS. dv-rejane@uol.com.br ${ }^{2}$ Coordenadoria Geral de Vigilância em Saúde, Equipe de Vigilância de Doenças Transmissíveis, Núcleo de Imunizações, $\mathrm{SMS} / \mathrm{POA} / \mathrm{RS}$

${ }^{3}$ Centro Estadual de Vigilância em Saúde (RS)

${ }^{4}$ Coordenadoria Geral de Vigilância em Saúde, Centro de Controle de Zoonoses, SMS/POA/RS
Abstract Animal bites are injuries that carry the risk of rabies transmission, a disease with a 100\% mortality rate. The purpose of this study was to determine the epidemiologic profiles of post exposure human anti-rabies treatments and to analyze whether prescriptions were appropriate. This cross-sectional study collected data from the forms of the Brazilian Notification System (Sistema Nacional Agravos de Notificação - SINAN), which were filled out by the professionals responsible for treatment in healthcare services in the second semester of 2006. Of the 2,223 cases identified, 50.3\% of the individuals were male, the age group with the greatest number of cases was 20 to 59 years (47.6\%); the type of injury responsible for the largest number of medical consultations was animal bite $(87.4 \%)$, and $35.3 \%$ of the injuries were in the lower extremities. Dogs were the animals that caused the most injuries (91.7\%). The analysis of type of treatment showed that vaccination was prescribed for $78.1 \%$ of the individuals, and antirabies serum, for $6.4 \%$. Of the all treatments, 96.2\% were classified as correctly prescribed. Although treatments were classified as necessary, the option of keeping animals that cause aggressions under observation should be considered so that the number of treatments administered can be reduced.

Key words Rabies, Epidemiologic surveillance, Epidemiologic profile
Resumo As mordeduras animais são injúrias que se destacam pela possibilidade de transmissão da raiva, sendo esta uma doença com letalidade de $100 \%$. O objetivo deste trabalho foi caracterizar o perfil epidemiológico do atendimento antirrábico humano pós-exposição e analisar a adequação de sua indicação. Foi realizado estudo transversal, tendo como fonte de dados a ficha de notificação do SINAN preenchida nos serviços de saúde pelos profissionais responsáveis pelo atendimento no segundo semestre de 2006. Entre os 2.223 casos investigados, 50,3\% dos individuos eram do sexo masculino; a faixa etária com mais agressões foi a de 20 a 59 anos (47,6\%); a forma de agressão responsável pela maior número de atendimentos foi a mordedura $(87,4 \%)$ e 35,3\% das agressões ocorreram nos membros inferiores. A espécie animal predominante foi a canina $(91,7 \%)$. Em relação ao tratamento prescrito, $78,1 \%$ dos sujeitos receberam indicação de vacina e $6,4 \%$, soro. Foram considerados como adequadamente indicados 96,2\% dos tratamentos. Embora os tratamentos tenham sido corretamente prescritos, faz-se necessária a valorização da observação do animal agressor para diminuir o número de tratamentos dispensados. Palavras-chave Raiva, Vigilância epidemiológica, Perfil epidemiológico 


\section{Introdução}

As injúrias são uma das principais causas de morbidade mundial, sobretudo na infância ${ }^{1}$. Entre elas, as agressões por animais, principalmente as caninas, são preocupantes pela possibilidade de transmissão da raiva e também pelos custos do tratamento ${ }^{1}$.

A cada ano, aproximadamente, quatro milhões de americanos são mordidos por cães, sendo metade desses agredidos pelo animal de parentes, vizinhos ou mesmo o seu ${ }^{2}$. Apesar disso, somente uma minoria procura atenção médica ${ }^{3}$. $\mathrm{Na}$ Bélgica, foi estimada uma prevalência anual de 22 agressões por cães para cada 1.000 jovens com menos de 15 anos no período entre 2001 e $2002^{4}$.

Os agravos provocados por mordeduras têm estado em evidência na mídia pelas agressões fatais a crianças e adultos, criando temor pelas raças ditas perigosas, como Rottweiler ou Pit Bull Terrier. No Brasil, segundo últimos dados disponíveis, ocorreram 16 óbitos por mordedura de cães em $2005^{5}$.

O Programa Nacional da Profilaxia da Raiva brasileiro foi criado em 1973, sendo implantado em todos os estados até 1977. Desde então, estão previstas ações de vigilância da população animal através de vacinação, captura e diagnóstico de laboratório; atendimento aos indivíduos agredidos e educação em saúde ${ }^{6}$. Os dados referentes à notificação de agravos provocados por animais são registrados na Ficha Individual de Notificação e digitados no Sistema de Informação de Agravos de Notificação - SINAN7 .

$A$ raiva não apresenta comportamento uniforme no Brasil. Em termos de casos humanos, é uma zoonose endêmica nas regiões Norte e Nordeste $^{8}$. No país, entre 2001 e 2006, ocorreram 110 casos de raiva, sendo $63,6 \%$ no sexo masculino. As faixas etárias mais atingidas foram a dos adultos, entre 20 a 39 anos (23,6\%), e jovens de 10 a 14 anos (22,7\%). A espécie agressora predominante foi a quiróptera, com $59 \mathrm{ca}-$ sos $(53,6 \%)$, seguida pela canina $(36,4 \%)^{9}$.

No Rio Grande do Sul, não são registrados casos de raiva humana desde 1981 e canina desde $1990^{10}$. No entanto, em 2005, foram atendidos para profilaxia antirrábica 20.270 pacientes, sendo os cães os principais agressores ${ }^{11}$.

Mesmo nos estados brasileiros onde a raiva está sob controle as ações de vigilância são necessárias ${ }^{12}$, pois é uma doença reemergente, para a qual não existe tratamento curativo após o início dos sintomas ${ }^{13}$. A indicação da vacina e o uso do soro são as estratégias utilizadas quando existe risco para a doença ${ }^{14}$.

A análise de dados referentes às mordeduras e à adequação do tratamento prescrito às normas técnicas vigentes é de extrema valia para a avaliação das ações de vigilância da raiva, humana e animal, das medidas de controle adotadas e da qualidade da assistência. Porém, no final de 2005, em Porto Alegre-RS, a Coordenadoria Geral de Vigilância em Saúde (CGVS) constatou problemas no preenchimento das fichas de atendimento antirrábico, dificultando a avaliação do programa. Com vistas a qualificar esse registro, foram realizadas capacitações dos profissionais responsáveis pelo atendimento nas unidades referência no primeiro semestre de 2006. Assim, o objetivo deste trabalho foi caracterizar o perfil epidemiológico do atendimento antirrábico humano pós-exposição e analisar a adequação de sua indicação, em Porto Alegre, no segundo semestre de 2006.

\section{Material e métodos}

Foi realizado um estudo transversal, investigando os 2.223 atendimentos antirrábicos humanos pós-exposição, realizados nas nove unidades referência para tratamento antirrábico humano em Porto Alegre, no segundo semestre de 2006.

A fonte de dados foi a ficha individual de notificação que é preenchida nos serviços de saúde de referência pelo técnico que realizou o atendimento e, após, enviada à Coordenadoria Geral de Vigilância em Saúde - CGVS/SMS para digitação no banco de dados do Sistema de Informação de Agravos de Notificação (SINAN).

As variáveis utilizadas para a caracterização do perfil epidemiológico das agressões foram relacionadas à pessoa agredida (sexo, idade, raça/ cor de pele e escolaridade), à agressão (local, tipo do ferimento, extensão do ferimento e tipo de exposição ao vírus), ao animal agressor (espécie e condição), ao tratamento (número de doses da vacina, uso de soro e tipo do soro). As idades foram agrupadas em faixas etárias, de acordo com os estágios de desenvolvimento humano, em crianças, adolescentes, adultos e idosos.

A partir dos dados disponíveis no sistema, foi criada uma variável denominada "gravidade”. De acordo com as Normas Técnicas para Tratamento Antirrábico Humano ${ }^{14}$, são consideradas agressões graves aquelas causadas por ferimentos localizados em mucosa, cabeça/pescoço, mãos/pés; ferimentos profundos, dilace- 
rantes ou múltiplos; ferimentos provocados por morcegos, animais silvestres ou de produção; ou provocados por cães e gatos suspeitos, desaparecidos, sacrificados ou mortos. Foi considerada como grave uma agressão que apresentasse, pelo menos, uma das características acima.

Os dados foram analisados utilizando os programas TabWin e Epi Info, sendo apresentados em relação à sua distribuição absoluta e relativa. Para testar as associações de interesse, foram utilizados o teste do qui-quadrado de associação e razão de prevalência com intervalo de confiança de $95 \%$ e nível de significância $<0,05$.

Para o estudo da adequação da indicação do tratamento, foram analisadas as informações constantes nas 2.223 fichas de atendimento, tomando-se como referência as Normas Técnicas para Tratamento Profilático Antirrábico Humano ${ }^{14}$. Nessa avaliação, para caninos e felinos, são consideradas as características do ferimento, o estado de saúde do animal no momento da agressão, possibilidade de observação do mesmo e situação epidemiológica local. Para os casos de contato com quirópteros ou agressão por animais de produção, a vacina deve ser sempre indicada.

Essas fichas foram, inicialmente, avaliadas por um veterinário da equipe de pesquisa. Aquelas que apresentaram discordância entre a prescrição do profissional de saúde e o preconizado pela norma técnica foram analisadas por um segundo avaliador, técnico da CGVS. Após, foram submetidas a uma terceira avaliação por especialista, sendo esta considerada como o padrão ouro. Ao final, os tratamentos foram classificados em adequadamente e inadequadamente indicados.

O presente estudo foi aprovado pelo Comitê de Ética e Pesquisa da Universidade Luterana do Brasil/RS, tendo a CGVS/SMS/POA consentido com o repasse dos dados.

\section{Resultados}

Durante o período de estudo, 57,7\% (1.282) dos 2.223 casos foram atendidos em uma única unidade referência, tendo os outros atendimentos se distribuído nas demais unidades.

Em relação à raça/cor da pele dos sujeitos agredidos, em 26,0\% (579) esse dado não foi preenchido. Excluindo-se os ignorados, encontrou-se 80,9\% (1.330/1.644) de indivíduos de cor branca, 10,9\% (179/1.644) preta, 7,5\% (124/1.644) parda, $0,5 \%$ (9/1.644) amarela e 0,1\% (2/1.644) indígena.

A escolaridade apresentou percentual mais alto de ignorados (50,7\%). Excluindo-se esses ca- sos, 3,6\% (40/1.096) não possuíam nenhuma escolaridade, 12,1\% (133/1.096) apresentavam de 1 a 3 anos de estudo concluído com êxito, 21,7\% (238/1.096) de 4 a 7 anos, 20,5\% (225/1.096) de 8 a 11 anos, $10,2 \%(112 / 1.096) 12$ ou mais anos e $31,7 \%$ (348/1.096) não estavam em idade escolar.

A distribuição entre os sexos foi bastante homogênea, com 50,3\% dos indivíduos pertencendo ao sexo masculino. Em dois casos, não foi possível resgatar esse dado, porém sabe-se que pertenciam à faixa etária de 20 a 59 anos (Tabela 1). A faixa etária, na qual ocorreram mais agressões, foi entre 20 e 59 anos, com 47,6\% dos casos (Tabela 1). Isoladamente, a idade que mais concentrou agressões foi a de 9 anos, com 71 casos, ocorrendo um predomínio entre os meninos $(60,6 \%)$.

Foi investigada a associação entre faixa etária e sexo, sendo verificada uma concentração de agressões no sexo feminino, em adultos (20 a 59 anos) e idosos ( $\geq 60$ anos). Por outro lado, entre crianças e adolescentes, o sexo masculino foi o mais agredido, sendo essas diferenças estatisticamente significativas $\left(\chi^{2}=55,13 ; \mathrm{p}=0,000\right)$.

A forma mais frequente de exposição ao vírus foi a mordedura e, em segundo lugar, a arranhadura. O ferimento foi profundo em mais da metade dos casos $(54,5 \%)$ e único em $64,7 \%$. A espécie animal agressora predominante foi a canina (Tabela 2).

Considerando, exclusivamente, as agressões provocadas pelas espécies canina e felina, crianças $(31,1 \%)$ e adolescentes $(10,4 \%)$ foram preponderantemente agredidos por cães e, adultos $(62,7 \%)$ e idosos $(23,7 \%)$, por felinos. Essas diferenças foram estatisticamente significativas $\left(\chi^{2}\right.$ $=57,25 ; \mathrm{p}=0,000)$.

Em relação ao sexo dos indivíduos agredidos, contatou-se que os homens sofreram mais

Tabela 1. Distribuição dos indivíduos atendidos para Tratamento Antirrábico Humano de acordo com sexo e faixa etária, Porto Alegre, RS, 2006.

\begin{tabular}{|c|c|c|c|c|c|c|}
\hline \multirow{3}{*}{ Faixa etária } & \multicolumn{4}{|c|}{ Sexo } & \multirow{2}{*}{\multicolumn{2}{|c|}{ Total }} \\
\hline & \multicolumn{2}{|c|}{ Masculino } & \multicolumn{2}{|c|}{ Feminino } & & \\
\hline & n & $\%$ & n & $\%$ & n & $\%$ \\
\hline 0 a 12 anos & 393 & 59,6 & 266 & 40,4 & 659 & 29,7 \\
\hline 13 a 19 anos & 124 & 57,9 & 90 & 42,1 & 214 & 9,6 \\
\hline 20 a 59 anos & 495 & 46,8 & 563 & 53,2 & 1.058 & 47,6 \\
\hline$\geq 60$ anos & 106 & 36,6 & 184 & 63,4 & 290 & 13,1 \\
\hline Total & 1.118 & 50,3 & 1.103 & 49,7 & 2.221 & 100,0 \\
\hline
\end{tabular}


agressões caninas $(51,8 \%)$, enquanto que as mulheres foram mais agredidas por felinos $(68,6 \%)$. A prevalência de agressões caninas foi $6 \%$ maior entre o sexo masculino do que no feminino (RP: 1,06; IC 95\%=1,04 - 1,09; $\mathrm{p}=0,000$ ).

Tabela 2. Distribuição dos indivíduos atendidos para Tratamento Antirrábico Humano de acordo com a exposição ao vírus, tipo e extensão do ferimento e espécie do animal agressor, Porto Alegre, RS, 2006.

\begin{tabular}{lrr}
\hline & $\mathbf{n}$ & $\%$ \\
\hline Exposição ao vírus & & \\
Mordedura & 2119 & 87,4 \\
Arranhadura & 281 & 11,6 \\
Lambedura & 15 & 0,6 \\
Contato indireto & 9 & 0,4 \\
Outro & 1 & 0,0 \\
Total & $\mathbf{2 4 2 2}$ & $\mathbf{1 0 0 , 0}$ \\
Tipo de ferimento & & \\
Profundo & 1233 & 54,5 \\
Superficial & 968 & 42,7 \\
Dilacerante & 63 & 2,8 \\
Total & $\mathbf{2 2 6 4}$ & $\mathbf{1 0 0 , 0}$ \\
Extensão do ferimento & & \\
Único & 1437 & 64,7 \\
Múltiplo & 723 & 32,5 \\
Ignorado & 63 & 2,8 \\
Espécie do animal agressor & & \\
Canina & 2039 & 91,7 \\
Felina & 169 & 7,7 \\
Quiróptera & 5 & 0,2 \\
Primata & 3 & 0,1 \\
Outra & 6 & 0,3 \\
Ignorada & 1 & 0,0 \\
Total & $\mathbf{2 2 2 3}$ & $\mathbf{1 0 0 , 0}$ \\
\hline
\end{tabular}

* Total superior ao número de pessoas atendidas, devido aos ferimentos múltiplos.
Em relação à localização, 35,3\% dos casos ocorreram nos membros inferiores e $32,2 \%$ nas mãos/pés. Analisando-se a distribuição dos indivíduos segundo a faixa etária e os locais de exposição, observou-se que entre as crianças e os adolescentes, os locais predominantes das agressões foram os membros inferiores. Para os indivíduos entre 20 e 59 anos, as mãos e pés e os membros inferiores tiveram praticamente a mesma frequência. Para os idosos, as lesões em mãos e pés foram prevalentes (Tabela 3 ).

Foram consideradas como graves $84,6 \%$ das agressões. Entre os homens, houve uma maior proporção de casos graves, apesar disso, essa diferença não foi estatisticamente significativa $\left(\chi^{2}=1,20 p=0,274\right)$. Da mesma forma, não houve associação entre gravidade e faixas etárias $\left(\chi^{2}=10,38 \mathrm{p}=0,065\right)$. No entanto, chama a atenção a ocorrência de cinco casos em crianças com menos de um ano, sendo todos esses casos considerados como graves. A prevalência de casos graves foi maior em crianças com menos de 5 anos (Tabela 4).

Quanto ao tratamento prescrito, $78,1 \%$ receberam indicação de vacina. Desses, para $77,4 \%$ (1.343/1.737) foram indicadas duas e 22,6\% (393) cinco doses. A indicação de soro ocorreu para apenas 6,4\% (112/1.737) dos indivíduos, sendo que, desses, 33,9\% (38) receberam soro homólogo, $11,6 \%$ (13) heterólogo e, em 54,5\% (61) dos casos, esse dado não foi preenchido pelo serviço de saúde.

Considerando exclusivamente a condição de observação de cães e gatos, identificou-se que a maioria dos atendimentos (986 casos) foi provocada por animais observáveis. Apesar disso, 80,9\% dos indivíduos receberam vacinas (Tabela 5).

Quanto à qualidade do atendimento, 96,2\% (1.672/1.737) das indicações de vacina e soro foram consideradas como adequadas e $98,6 \%$ (478/ 486) dos casos de não indicação também foram

Tabela 3. Distribuição dos indivíduos atendidos para Tratamento Antirrábico Humano de acordo com a faixa etária e locais de exposição, Porto Alegre, RS, 2006

\begin{tabular}{|c|c|c|c|c|c|c|c|c|c|c|c|c|c|c|}
\hline \multirow{3}{*}{$\begin{array}{c}\text { Faixa etária } \\
\text { (em anos) }\end{array}$} & \multicolumn{14}{|c|}{ Locais de exposição } \\
\hline & \multicolumn{2}{|c|}{ Mucosa } & \multicolumn{2}{|c|}{ Cabeça } & \multicolumn{2}{|c|}{ Mãos/pés } & \multicolumn{2}{|c|}{ Tronco } & \multicolumn{2}{|c|}{$\begin{array}{l}\text { Membros } \\
\text { superiores }\end{array}$} & \multicolumn{2}{|c|}{$\begin{array}{l}\text { Membros } \\
\text { inferiores }\end{array}$} & \multicolumn{2}{|c|}{ Total } \\
\hline & n & $\%$ & n & $\%$ & $\mathbf{n}$ & $\%$ & $\mathbf{n}$ & $\%$ & n & $\%$ & $\mathbf{n}$ & $\%$ & $\mathbf{n}$ & $\%$ \\
\hline$<13$ & 22 & 31 & 174 & 24,3 & 138 & 19,3 & 70 & 9,8 & 90 & 12,6 & 221 & 30,9 & 715 & 29,8 \\
\hline 13 a 19 anos & 2 & 0,9 & 14 & 6,1 & 57 & 24,8 & 15 & 6,5 & 40 & 17,4 & 102 & 44,3 & 230 & 9,6 \\
\hline 20 a 59 anos & 4 & 0,3 & 51 & 4,5 & 433 & 37,8 & 43 & 3,8 & 184 & 16,1 & 429 & 37,5 & 1144 & 47,7 \\
\hline$\geq 60$ anos & 0 & 0,0 & 7 & 2,3 & 144 & 46,4 & 7 & 2,3 & 57 & 18,4 & 95 & 30,6 & 310 & 12,9 \\
\hline Total & 28 & 1,2 & 246 & 10,2 & 772 & 32,2 & 135 & 5,6 & 371 & 15,5 & 847 & 35,3 & 2399 & 100,0 \\
\hline
\end{tabular}


adequados. Por fim, em relação à conclusão do tratamento, segundo registros do SINAN, 55,4\% (962/1.737) dos indivíduos foram identificados como tendo interrompido o tratamento vacinal indicado.

\section{Discussão}

O estudo do perfil epidemiológico das agressões possibilita o conhecimento das situações envolvidas, servindo de base para a definição de estratégias de prevenção, controle e avaliação da raiva humana ${ }^{15}$. Este estudo poderá servir de base para futuros estudos analíticos.

Foi observada uma distribuição desigual dos atendimentos, com maior concentração em uma unidade referência. Uma das razões para que isso ocorra pode ser a maior disponibilidade de atendimento nessa unidade, ocorrendo na forma de

Tabela 4. Distribuição dos indivíduos atendidos para Tratamento Antirrábico Humano de acordo com a gravidade em relação ao sexo e faixa etária, Porto Alegre, RS, 2006

\begin{tabular}{lrrrrr}
\hline & \multicolumn{2}{c}{ Sim } & & \multicolumn{2}{c}{ Não } \\
\cline { 2 - 3 } \cline { 5 - 6 } & $\mathbf{n}$ & $\%$ & & $\mathbf{n}$ & $\%$ \\
\hline Sexo & & & & & \\
$\quad$ feminino & 923 & 83,8 & & 179 & 16,2 \\
$\quad$ masculino & 956 & 85,4 & & 163 & 14,6 \\
& 1.879 & 84,6 & & 342 & 15,4 \\
Faixa etária & & & & & \\
$\quad<1$ anos & 5 & 100,0 & & - & - \\
1 a 5 anos & 209 & 86,7 & & 32 & 13,3 \\
6 a 12 anos & 336 & 81,2 & & 78 & 18,8 \\
13 a 19 anos & 171 & 80,3 & & 42 & 19,7 \\
20 a 59 anos & 911 & 85,9 & & 149 & 14,1 \\
$\quad$ 60 anos & 249 & 85,9 & & 41 & 14,1 \\
Total & $\mathbf{1 . 8 8 1}$ & $\mathbf{8 4 , 6}$ & & $\mathbf{3 4 2}$ & $\mathbf{1 5 , 4}$ \\
\hline
\end{tabular}

plantões diários, de 24 horas, inclusive em feriados. Além disso, esse serviço é o único responsável pela aplicação de soro nos casos considerados de maior gravidade em adultos. Assim, verifica-se a necessidade da facilitação do acesso às outras unidades referência, ampliando o horário de atendimento e divulgando o serviço.

Apesar da capacitação realizada, alguns dados ainda apresentaram elevado percentual de respostas ignoradas, como foi o caso da raça/ cor da pele e escolaridade, dificultando a caracterização do perfil dos indivíduos de maneira adequada. Nesse sentido, é importante que os serviços responsáveis pela coordenação do Programa de Profilaxia e Controle da Raiva invistam esforços na sensibilização e na capacitação dos serviços de referência para o correto e completo preenchimento das fichas do SINAN.

Em relação aos casos de raiva no $\mathrm{Brasil}^{16}$, $74,9 \%$ ocorreram no sexo masculino. Nas Américas $^{17-21}$, o percentual de homens que desenvolveram a doença variou de $61,0 \%{ }^{19}$ a $72,2 \%{ }^{17}$. No presente estudo, houve homogeneidade na distribuição das agressões entre os sexos. No entanto, alguns autores identificaram de leve $e^{22,23}$ a marcante ${ }^{12,15,24-27}$ predomínio no sexo masculino, chegando a $69,4 \%$ em Nova Delhi ${ }^{28}$.

Entre o sexo masculino, as agressões ocorreram mais na infância e na adolescência, e entre o feminino, acima dos 20 anos. Essa situação também foi descrita por outros autores ${ }^{29,30}$. No município de Jacarezinho, Paraná ${ }^{27}$, e na Grande São Paulo $^{31}$, foi constatada maior exposição de meninos entre 5 e 9 anos. Supõe-se que o sexo masculino tenha maior risco de agressões por cães e, consequentemente, de contrair a raiva, devido ao fato de terem maior contato com os animais, estarem mais tempo fora de casa, tendo atitudes e brincadeiras que podem estimular à agressão ${ }^{18,27}$. As meninas, em contrapartida, costumam desenvolver brincadeiras dentro do domicílio, expondo-se menos às agressões.

Tabela 5. Distribuição dos indivíduos atendidos para Tratamento Antirrábico Humano de acordo com a condição do animal agressor (somente caninos e felinos) e indicação do tratamento, Porto Alegre, RS, 2006

\begin{tabular}{|c|c|c|c|c|c|c|c|c|c|c|c|c|c|c|}
\hline & \multicolumn{14}{|c|}{ Condição do animal agressor } \\
\hline & \multicolumn{2}{|c|}{ Sadio } & \multicolumn{2}{|c|}{ Suspeito } & \multicolumn{2}{|c|}{ Desaparecido } & \multicolumn{2}{|c|}{ Observável } & \multicolumn{2}{|c|}{ Sacrificado } & \multicolumn{2}{|c|}{ Ignorado } & \multicolumn{2}{|c|}{ Total } \\
\hline & $\mathbf{n}$ & $\%$ & $\mathbf{n}$ & $\%$ & $\mathbf{n}$ & $\%$ & $\mathbf{n}$ & $\%$ & $\mathbf{n}$ & $\%$ & $\mathbf{n}$ & $\%$ & $\mathbf{n}$ & $\%$ \\
\hline \multicolumn{15}{|c|}{ Vacinação } \\
\hline Sim & 554 & 60,0 & 24 & 96,0 & 188 & 98,4 & 798 & 80,9 & 39 & 95,1 & 134 & 95,7 & 1737 & 78,1 \\
\hline Não & 286 & 34,0 & 1 & 4,0 & 3 & 1,6 & 188 & 19,1 & 2 & 4,9 & 6 & 4,3 & 486 & 21,9 \\
\hline Total & 840 & 100,0 & 25 & 100,0 & 191 & 100,0 & 986 & 100,0 & 41 & 100,0 & 140 & 100,0 & 2223 & 100,0 \\
\hline
\end{tabular}


Em Porto Alegre, houve um maior número de atendimentos de indivíduos adultos. Diferente disso, vários estudos ${ }^{1,12,15,24,25,31}$ relataram que as crianças foram o grupo populacional mais atendido. É possível que na cidade aqui estudada os adultos estejam mais expostos a agressões animais em suas atividades diárias, como, por exemplo, trabalho, cuidado com os animais domésticos e deslocamento pelas vias públicas.

Entre os adultos, as mulheres foram mais agredidas por animais, em especial, as idosas, devido ao manuseio e cuidados com seus animais domésticos ${ }^{27}$.

O tipo de agravo responsável pelo maior número de atendimentos foi a mordedura, semelhante a outros estudos realizados no país ${ }^{1,12,23-}$ 25,31,32. É possível que o alto percentual de mordeduras entre os casos atendidos deva-se ao conhecimento da população sobre o risco deste tipo de exposição para a raiva, o que não ocorre com os outros tipos de exposição ${ }^{31}$, em especial com a lambedura ou a arranhadura. Esse dado pode não corresponder ao total das agressões ocorridas, pois se refere apenas às registradas no SINAN, representando aqueles indivíduos que procuram o serviço.

O ferimento profundo ocorreu em mais da metade das agressões, havendo predominância de ferimentos únicos. Carvalho e Silva ${ }^{15}$ também identificaram 77\% de lesões profundas e Carvalho et al. ${ }^{12}$ encontraram $61,1 \%$ ferimentos únicos. Essa predominância de ferimentos profundos pode indicar que, quando as pessoas procuram atendimento, o fazem preferencialmente para os casos de agressões mais graves.

A espécie canina foi responsável por $91,7 \%$ das situações de busca de atendimento. Em outros estudos $8,12,23,25-27,32$, também houve o predomínio desta espécie, variando de $83,7 \%{ }^{28}$, em Nova Delhi, a $91,3 \%{ }^{33}$, na $5^{\text {a }}$ Coordenadoria Regional de Saúde, Caxias do Sul. Diferentes resultados foram encontrados em estudo realizado, em Nova Iorque, que demonstrou que $49 \%$ dos atendimentos foram em decorrência de agressões por animais selvagens, sendo os quirópteros responsáveis por $61 \%$ desses atendimentos ${ }^{16}$. Ao contrário do que ocorre com os caninos, no caso dos quirópteros, as agressões são habitualmente acidentais.

Com relação às agressões provocadas pelos cães, as crianças e os adolescentes foram os mais agredidos, assim como os indivíduos do sexo masculino. Quanto à espécie felina, as faixas etárias preferenciais foram a dos adultos e idosos e o sexo feminino. Patrick e O'Rourke ${ }^{30}$, investigando as vítimas das agressões de cães em El Paso,
Texas, identificaram que $61,1 \%$ eram do sexo masculino, sendo a maioria crianças e adolescentes; enquanto que, entre as vítimas das agressões de felinos, $57,5 \%$ eram do sexo feminino, predominando adultos e idosos. As mulheres são mais agredidas na fase adulta, principalmente por felinos, possivelmente devido a seu estreito cuidado com os animais domésticos, resultando agressões nas mãos, conforme nossos achados.

A localização preferencial das agressões foi nos membros inferiores, assemelhando-se ao relatado por outros autores ${ }^{5,6,15,26}$, que encontraram de $30,4 \%{ }^{32}$ a $55 \%{ }^{15}$. Em crianças e adolescentes, o local mais atingido foi o membro inferior. Esse local é mais acessível para os animais, sendo sede de um grande número de agressões ${ }^{23}$. Del Ciampo et al. ${ }^{1}$ também encontraram, nesse mesmo grupo etário, os membros inferiores como sendo os locais mais atingidos. Diferente disso, Rolim et al. ${ }^{27}$ constataram que mordeduras na cabeça/pescoço ocorrem mais em crianças de até nove anos. Entretanto, nesse mesmo estudo, para os adolescentes, o local de maior agressão foram os membros inferiores concordando com os achados de Porto Alegre. Alonso ${ }^{23}$ também refere que, em crianças de até quatro anos, predominam as agressões na cabeça/pescoço, porém, entre os 5 e 14 anos, os locais mais frequentes são os membros inferiores e, após os 15 anos, as mãos e os pés.

Neste estudo, na faixa etária entre 20 e 59 anos, os locais mais atingidos foram as mãos/ pés, seguidos pelos membros inferiores e, para os idosos, foram as mãos/pés. Segundo Rolim et al. ${ }^{27}$, em indivíduos com 50 anos ou mais anos, as mulheres são mais suscetíveis à agressão com localização nas mãos. É possível que isso ocorra em função das pessoas com mais idade serem agredidas por seus próprios animais, durante o cuidado dos mesmos. Enquanto que os mais jovens são agredidos por animais na rua ou enquanto trabalham.

Em relação à gravidade da lesão, a maioria foi classificada como grave, percentual superior ao encontrado em Campo Grande, $\mathrm{MS}^{32}$. Conforme já discutido anteriormente, esse resultado pode sugerir que a população procura o atendimento, preferencialmente, para esse tipo de lesões. Porém, é importante ressaltar que quando o animal encontra-se infectado pelo vírus rábico e causa uma lesão única e superficial, a letalidade é de $35 \%{ }^{16}$. Esse dado é importante, pois reforça a necessidade da procura pelo atendimento antirrábico sempre que houver uma possível exposição ao vírus, para que seja realizada avaliação e acompanhamento do caso pelo serviço de saúde. 
A avaliação da qualidade do tratamento indicado foi realizada do ponto de vista quantitativo e qualitativo. Quanto ao aspecto quantitativo, das pessoas que procuraram o atendimento, $78,1 \%$ receberam indicação de vacina e apenas uma pequena parcela também recebeu soro. Outros estudos também demonstram altos percentuais de tratamento ${ }^{1,33}$ e baixa utilização de soro $^{33}$. Diferente do presente estudo, foram encontrados trabalhos com menores percentuais de tratamento, como no estado de São Paulo $(8,70 \%)^{23}$, Maringá, no Paraná $(40,9 \%)^{12}$, e na Grande São Paulo $(49,4 \%)^{31}$. O elevado número de pessoas tratadas em relação às atendidas nos leva a pensar que muitos tratamentos poderiam ser evitados com a observação do animal agressor por $10 \mathrm{dias}^{34}$. Com esse procedimento, a profilaxia humana seria indicada somente se o animal manifestasse sintomas da doença ${ }^{25}$. No presente estudo, evidenciou-se que a população procura o atendimento quando a agressão é grave, o que também contribui para o elevado número da indicação de vacinas.

A tese da prescrição excessiva de vacinas é comprovada pelo alto percentual de indivíduos $(80,9 \%)$ que foram agredidos por animais observáveis que, mesmo assim, receberam indicação de vacina antirrábica. Por meio da observação do animal e o mesmo permanecendo sadio no período recomendado, previne-se a prescrição desnecessária de tratamentos, diminuindo a possibilidade de reações adversas e gastos públicos ${ }^{23}$.

Ao seguir a Norma Técnica, sem uma análise mais particularizada do caso, levando em consideração a observação do animal e a condição epidemiológica da raiva na região, o profissional é levado à indicação da vacina. Esse procedimento é correto, entretanto, expõe o paciente a riscos desnecessários. A Norma Técnica, em sua nota de rodapé, esclarece que se o animal agressor for exclusivamente domiciliado e sem contato com outros animais, o agredido poderá ser dispensado do tratamento, desde que o animal seja observado. Essa observação deve ser de qualidade, ou seja, realizada por profissional qualificado.

Um aspecto referente à qualidade do tratamento prescrito, diz respeito ao alto percentual de indicação correta $(96,2 \%)$, segundo as Normas Técnicas, e, mais alto ainda, de adequação da não indicação (98,6\%). Esses resultados são mais positivos do que os encontrados em outros estudos. Em Mato Grosso do Sul, 41,9\% dos tratamentos foram classificados como adequadamente indicados, $24,7 \%$ como inadequados, $29,9 \%$ possuíam dados insuficientes e $3,6 \%$ não foram classificados ${ }^{32}$. Estudo realizado nos Estados Unidos apresentou 40,0\% de inadequação de indicação de tratamento e $6,3 \%$ de inadequação para os que foram dispensados desse. Foram apontados como motivos da inadequação o animal estar disponível para a observação ou teste e prescrições em zonas de baixa endemicidade ${ }^{35}$.

A região de procedência do animal deve ser avaliada no momento da prescrição do tratamento, pois se o local é de alta endemicidade, o tratamento deve ser instituído. No entanto, mesmo em outras áreas, cada caso deve ser avaliado cuidadosamente, segundo as normas técnicas. Além da avaliação da situação epidemiológica da região da procedência do animal, são decisivos para a adequada condução do caso: a integração dos serviços de atendimento médico e médico veterinário, a análise das características da agressão e a avaliação do animal agressor ${ }^{36}$.

Quanto à conclusão do tratamento, mais da metade dos sujeitos foram considerados como tendo interrompido o mesmo. É possível que esses valores não sejam tão elevados, pois os indivíduos, muitas vezes, trocam de unidade referência para a continuidade da vacina. Nessas situações, o serviço que iniciou o tratamento pode não ser informado, não sendo registrados nas fichas de Atendimento Antirrábico Humano os dados referentes à conclusão do esquema vacinal. Caso as fichas não contenham dados atualizados, haverá perda da qualidade das informações produzidas a partir do SINAN.

Melhores resultados em relação ao abandono foram encontrados em Maringá, PR $(4,9 \%)^{12}$, no estado de São Paulo $(10,3 \%)^{25}$ e no RS $(14,7 \%)^{33}$.

\section{Considerações finais}

A raiva é uma doença sob controle no Brasil. Entretanto, nos últimos anos, ainda têm sido evidenciado óbitos humanos pela doenç ${ }^{37}$. Em função disso, é imprescindível que se intensifique as medidas de prevenção. Nesse sentido, acredita-se que existam quatro estratégias fundamentais a serem implementadas: 1) vigilância de animais e da saúde humana; 2) educação em saúde; 3) posse responsável de animais e 4) qualificação do sistema de informações.

A vigilância animal inclui a coleta de material para diagnóstico, a vacinação e a observação qualificada dos animais agressores ${ }^{38}$, podendo ser realizada no nível municipal, tanto de forma centralizada como descentralizada, na dependência 
da existência de recursos locais e da distribuição geográfica dos serviços básicos de saúde.

A vigilância da saúde humana é baseada no atendimento dos indivíduos expostos a agressões animais. Para o estabelecimento da conduta terapêutica adequada, é necessário avaliar o tipo de ferimento, as circunstâncias da agressão, a avaliação do animal agressor, a condição epidemiológica do território (circulação viral) e, em casos de cães e gatos, a possibilidade de observação animal, devendo ser, cada caso, avaliado individualmente. Para reduzir a profilaxia desnecessária, deve haver um aumento da observação dos animais e a integração entre os serviços de saúde médico e médico veterinário. Quanto mais profissionais forem capacitados para fazerem parte dessa rede de vigilância, mais sucesso terão as estratégias de prevenção, controle e tratamento da raiva.

Em relação à educação em saúde, essa diz respeito tanto aos profissionais quanto à população em geral. É necessária a capacitação da equipe de saúde para o completo preenchimento das fichas individuais de atendimento e a responsabilização pela busca ativa dos faltosos ao tratamento. A população deve ser orientada por veterinários e profissionais de cuidados primários sobre o comportamento dos animais ${ }^{2}$ e medidas frente às agressões.

A posse responsável é baseada nas ações para garantir a convivência saudável entre pessoas e animais. Estimular a posse responsável é de extrema importância para evitar animais em "situação de rua", o aumento da população animal e a disseminação de doenças.

Por fim, para se reduzir o sub-registro referente à conclusão do tratamento, é necessário que o SINAN seja disponibilizado on line, em intranet, de forma que qualquer serviço de referência que realize a aplicação da vacina possa informar que o paciente recebeu a dose aprazada, logo após o procedimento.

Ainda que isoladamente essas estratégias sejam bastante efetivas, só a ação simultânea poderá garantir o adequado enfrentamento dessa gravíssima zoonose.

\section{Colaboradores}

RD Veloso foi responsável pela concepção do estudo, coleta de dados e redação do artigo. DRGC Aerts participou da concepção do estudo, supervisão da coleta de dados e redação do artigo. LO Fetzer auxiliou na concepção do estudo e participou da redação final do artigo. CB dos Anjos realizou a revisão final do artigo. JC Sangiovanni participou da avaliação da qualidade do atendimento antirrábico humano e revisão final do artigo. 


\section{Referências}

1. Del Ciampo, LA, Ricco, RG, Almeida CA de, Bonilha LRCM, Santos TCC. Acidentes de mordeduras de cães na infância. Rev Saude Publica 2000; 34(4): 411-412.

2. Presutti RJ. Prevention and treatment of dogs bites. Am Fam Physician 2001; 63(8):1567-1572.

3. Morgan M, Palmer J. Dog bites. BMJ 2007; 334(7590):413-417.

4. De Keuster T, Lamoureux J, Kahn, A. Epidemiology of dog bites: A Belgian experience of canine behaviour and public health concerns. Vet J 2006; 172(3):482-487.

5. Brasil. Ministério da Saúde (MS). Óbitos por mordedura ou golpe provocados por cão - Brasil. [acessado 2007 nov 10]. Disponível em: http://tabnet.datasus. gov.br/cgi/deftohtm.exe?sim/cnv/extuf.def

6. Schneider MC, Almeida GA de, Souza LM, Morares NB de, Diaz RC. Controle da raiva no Brasil de 1980 a 1990. Rev Saude Publica 1996; 30(2):196-203.

7. Brasil. Ministério da Saúde (MS). Sistema de Informação de Agravos de Notificação - SINAN. [acessado 2007 nov 11]. Disponível em: http://www.saude. gov.br/saude/visualizar_texto.cfm?idtxt $=21383$

8. Brasil. Ministério da Saúde (MS). Raiva humana Distribuição de casos confirmados, por Unidade Federativa, Brasil, 1980-2005. [acessado 2007 nov 12]. Disponível em: http://dtr2004.saude.gov.br/ sinanweb/tabnet/tabnet?sinan//raiva/bases/ raivabr.defwww.saude.gov.br

9. Brasil. Ministério da Saúde (MS). Raiva - Casos confirmados notificados no Sistema de Informação de Agravos de Notificação - SINAN, segundo sexo, faixa etária, idade e espécie agressora. [acessado 2007 nov 19]. Disponível em: http://dtr2004. saude.gov.br/sinanweb/tabnet/tabnet?sinan//raiva/ bases/raivabr.defwww.saude.gov.br

10. Rio Grande do Sul. Secretaria Estadual da Saúde (SES). RS registra 24 casos de raiva transmitida por morcegos em 2006. [acessado 2006 ago 5]. Disponível em: http://www.saude.rs.gov.br/noticia_view. php?id_noticia $=1708$

11. Rio Grande do Sul. Secretaria Estadual da Saúde (SES). Centro Estadual de Vigilância em Saúde (CEVS). Rede Estadual de Análise e Divulgação de Indicadores para a Saúde. A saúde da população do estado do Rio Grande do Sul. Porto Alegre: CEVS; 2006.

12. Carvalho WO, Soares DFP de P, Franceschi VCS. Características do atendimento prestado pelo serviço de profilaxia da raiva humana na rede municipal de Saúde de Maringá-Paraná, no ano de 1997. Inf Epidemiol SUS 2002; 11(1):25-35.

13. Vera C, Vera L, Terreros MC. Mordeduras de perros: Tratamiento, Complicaciones y Prevención. Pediatr Dia 2004; 20(1):30-35.

14. Brasil. Ministério da Saúde (MS). Normas Técnicas de Tratamento Profilático Antirrábico Humano. Brasília: Ministério da Saúde (MS); 2002.

15. Carvalho CC, Silva BTF. Características epidemiológicas de acidentes por mordedura de cão atendidos em unidade básica de saúde no nordeste do Brasil. RBPS 2007; 20(1):17-21.
16. Carrieri ML, Takaoka NY, Kotait I, Germano PML Diagnóstico clínico-epidemiológico da raiva humana: dados do Instituto Pasteur de São Paulo do período de 1970-2002. BEPA 2006; 29:2-8.

17. Centro Panamericano de Fiebre Aftosa. Boletín de Vigilância Epidemiológica de La Rabia em las Américas, 2000; XXX I I:10-2. [acessado 2008 mai 24]. Disponível em: http://bvs.panaftosa.org.br/textoc/ bolvera2000.pdf

18. Centro Panamericano de Fiebre Aftosa. Boletín de Vigilância Epidemiológica de La Rabia em las Américas, 2001; XXX I I I:12. [acessado 2008 mai 24]. Disponível em: http://bvs.panaftosa.org.br/textoc/ bolvera2001.pdf

19. Centro Panamericano de Fiebre Aftosa. Boletín de Vigilância Epidemiológica de La Rabia em las Américas, 2002; XXXIV: 15. [acessado 2008 mai 24]. Disponível em: http://bvs.panaftosa.org.br/textoc/ bolvera2002.pdf

20. Centro Panamericano de Fiebre Aftosa. Boletín de Vigilância Epidemiológica de La Rabia em las Américas, 2003; XXXV: 11. [acessado 2008 mai 24]. Disponível em: http://bvs.panaftosa.org.br/textoc/ bolvera2003.pdf

21. Centro Panamericano de Fiebre Aftosa. Boletín de Vigilância Epidemiológica de La Rabia em las Américas, 2004; XXXVI: 11. [acessado 2008 mai 24]. Disponível em: http://bvs.panaftosa.org.br/textoc/ bolvera2004.pdf

22. Blanton JD, Bowden NY, Eidson M, Wyatt JD, Hanlon CA. Rabies postexposure prophylaxis, New York, 1995-2000. Emerg Infect Dis 2005; 11(12):1921-1927.

23. Alonso BPM. Estudo dos casos de agressões por cães no município de Araraquara, estado de São Paulo, Brasil [monografia]. Araraquara: Faculdade de Ciências Farmacêuticas da Universidade Estadual Paulista Júlio de Mesquita Filho; 2005.

24. Lippolis M, Dutra Sobrinho JP, Benites NR. Epidemiologia e avaliação dos fatores de risco associados a acidentes por mordedura de cães em humanos, no município de Guarulhos, estado de São Paulo, de 1997-2003. Arq Inst Biol 2004; 71(Supl.): 375-377.

25. Pinto CL, Alleoni ES. Aspectos da vigilância epidemiológica da raiva em sub-regiões administrativas do Estado de São Paulo, Brasil, 1982-1983. Rev Saude Publica 1986; 20(4):288-292.

26. Ribeiro Netto A, Machado CG. Alguns aspectos epidemiológicos da exposição humana ao risco da infecção pelo vírus da raiva, na cidade de São Paulo, Brasil. Rev Inst Med Trop 1970; 12(1):16-30.

27. Rolim RLP, Lopes FMR, Navarro IT. Aspectos da vigilância epidemiológica no município de Jacarezinho, Paraná, Brasil, 2003. Semina: Ciências Agrárias $2006 ; 27(2): 271-280$.

28. Rasania SK, Bhalla S, Khandekar J, Pathi S, Matta S, Singh S. Post exposure management of animal bite cases attending a primary health center of Delhi. J Commun Dis 2004; 36(3):195-198.

29. Vera C, Vera L, Terreros MC. Mordeduras de perros: Tratamiento, complicaciones y prevención. $P e$ diatr Dia 2004; 20(1):30-35. 
30. Patrick GR, O'Rourke KM. Dog and cat bites: epidemiologic analyses suggest different prevention strategies. Public Health Rep 1998; 113(3):252-257.

31. Garcia RCM, Vasconcellos AS, Sakamoto SM, Lopez AC. Análise de tratamento antirrábico humano pós-exposição em região da Grande São Paulo, Brasil. Rev Saude Publica 1999; 33(3):295-301.

32. Rigo L, Honer MR. Análise da profilaxia da raiva humana em Campo Grande, Mato Grosso do Sul, Brasil, em 2002. Cad Saude Publica 2005; 21(6):19391945.

33. Rio Grande do Sul. Secretaria Estadual da Saúde Centro Estadual de Vigilância em Saúde (SES/CEVS). Boletim Epidemiológico 2005; 7(3):1-4.

34. Pereira Filho M, Silva LMC. Aspectos Epidemiológicos da Raiva no Estado da Bahia período: 1979 a 1980. Rev Baiana Saúde Públ 1982; 9(1):16-19.

35. Moran GJ, Talan DA, Mower W, Newdow M, Ong S, Nakase JY, Pinner RW, Childs JE. Appropriateness of rabies postexposure prophylaxis treatment for animal exposures. JAMA 2000; 284(8):1001-1007.

36. Costa WA, Ávila CA, Valentine EJG, Reichmann MLAB, Cunha RS, Guidolin R, Panachão MRI, Omoto TM, Bolzan VL. Profilaxia da raiva humana. 2.ed. São Paulo: Instituto Pasteur; 2000.

37. Brasil. Ministério da Saúde (MS). Óbitos da raiva humana, segundo UF de residência. Brasil, Grandes regiões e unidades federativas, 1990 a 2008. [acessado 2009 jul 26]. Disponível em: http://portal. saude.gov.br/portal/arquivos/pdf/OBITOS_RAIVA. pdf

38. Brasil. Ministério da Saúde (MS). Secretaria de Vigilância em Saúde. Guia de Vigilância Epidemiológica. 6a ed. Brasília: Ministério da Saúde (MS); 2005.

Artigo apresentado em 19/06/2009

Aprovado em 23/01/2010

Versão final apresentada em 05/02/2010 